\title{
Bistable nerve conduction
}

\author{
Zhaoyang Zhang ${ }^{1}$, Zhilin $\mathrm{Qu}^{1,2, *}$ \\ ${ }^{1}$ Department of Medicine and ${ }^{2}$ Department of Computational Medicine, David Geffen School of \\ Medicine, University of California, Los Angeles, California 90095, USA
}

Short title: Bistable nerve conduction

*Correspondence to:

Zhilin Qu, PhD

Department of Medicine

Division of Cardiology

David Geffen School of Medicine at UCLA

A2-237 CHS, 650 Charles E. Young Drive South

Los Angeles, CA 90095

Email: zqu@mednet.ucla.edu 


\begin{abstract}
The essence of the nerve system is to transmit information from and to different parts of the body in response to environmental changes. Here we show that a bistable conduction behavior can occur in the nerve system, which exhibits stimulus-dependent fast and slow conduction waves. The bistable behavior is caused by a positive feedback loop of the wavefront upstroke speed, mediated by the sodium channel inactivation properties, which is further potentiated by the calcium current. This provides a mechanism for the slow and fast conduction in the same nerve system observed experimentally. We show that the bistable conduction behavior is robust with respect to the experimentally determined activation thresholds of the known sodium and calcium channel families. The theoretical insights provide a generic mechanism for stimulus-dependent fast and slow conduction in the nerve system, which is applicable to conduction in other electrically excitable tissue, such as cardiac muscles.
\end{abstract}




\section{Introduction}

The major function of the nerve system is to transmit information via electrical excitation and conduction for the body or parts of the body to respond to environmental changes. The response time is determined by the conduction velocity (CV) of the nerve cable. It is well established that $\mathrm{CV}$ is determined by the conductance of the sodium $\left(\mathrm{Na}^{+}\right)$current $\left(\mathrm{I}_{\mathrm{Na}}\right)$ and calcium $\left(\mathrm{Ca}^{2+}\right)$ current $\left(\mathrm{I}_{\mathrm{Ca}}\right)$, the size of the nerve cable, temperature, and myelination, etc ${ }^{1-4} . \mathrm{CV}$ in different nerve fibers or species spans in a wide range, differing in orders of magnitude ${ }^{1}$. These conduction properties can be well described by the cable equation with the Hodgkin-Huxley (HH) model ${ }^{5-10}$.

Besides the regular conduction properties, an interesting conduction behavior was observed experimentally, i.e., stimulus-dependent fast and slow conduction waves occur in the same nerve fiber. For example, Aglantha digitale, a species of jellyfish, has two modes of swimming - a slow swimming for fishing and a fast swimming for escaping away from predators ${ }^{11,12}$. In an experimental study ${ }^{13}$, Mackie and Meech showed that the slow and fast swimming modes were caused by a slow conduction $(\sim 0.3 \mathrm{~m} / \mathrm{s})$ and a fast conduction $(\sim 1.4 \mathrm{~m} / \mathrm{s})$ in the same motor giant axon, respectively. The slow conduction is a low-amplitude and long-duration wave mediated by $\mathrm{I}_{\mathrm{Ca}}$ and the fast conduction is a high-amplitude and short-duration wave mediated by $\mathrm{I}_{\mathrm{Na}}$ (Fig.S1). The fast wave was induced by an external stimulus and the slow wave was spontaneous and endogenous. This same conduction dynamics was also shown in experiments of conduction in cockroach giant axons by Hochner and Spira ${ }^{14}$ who demonstrated that two distinct conduction waves $(0.1-0.6 \mathrm{~m} / \mathrm{s}$ and $3-6 \mathrm{~m} / \mathrm{s})$ occurred in the same axon treated with ethanol. Differing from Aglantha digitale, the slow conduction in the cockroach giant axon was not mediated by $\mathrm{I}_{\mathrm{Ca}}$ but still by $\mathrm{I}_{\mathrm{Na}}$. Besides conduction in the giant axons of jellyfish and cockroaches, evidence of stimulus-dependent fast and slow conduction has also been shown in experiments of rat visual cortex ${ }^{15,16}$, i.e., the $\mathrm{CV}$ of the spontaneous waves differ from those of the evoked waves in the same cortex. These experimental observations imply that besides the regular conduction behavior, a new conduction dynamics can occur in the nerve systems, i.e., the same nerve fiber or tissue can exhibit two stable conduction states depending on the initial conditions. As in Aglantha digitale, the two conduction behaviors of the same fiber accomplish two distinct biological functions, however, the underlying mechanism remains to be elucidated.

In this study, we investigate the mechanisms of the stimulus-dependent fast and slow conduction using analytical methods and computer simulations in a cable equation with the $\mathrm{HH}$ model ${ }^{17}$. An $\mathrm{I}_{\mathrm{Ca}}$ formulation is added to the $\mathrm{HH}$ model to investigate the role of $\mathrm{I}_{\mathrm{Ca}}$. We show that stimulus-dependent fast and slow conduction capturing the experimental observations can occur in the cable with the $\mathrm{HH}$ model. This is a bistable behavior, namely bistable conduction, emerging during conduction in the cable, caused by a positive feedback loop of the wavefront upstroke speed mediated by the $\mathrm{Na}^{+}$channel inactivation properties. The addition of $\mathrm{I}_{\mathrm{Ca}}$ can further potentiate bistable conduction. Using simulations of randomly selected parameter sets, we show that the bistable conduction mechanism is robust, i.e., the activation thresholds of $\mathrm{I}_{\mathrm{Na}}$ and $\mathrm{I}_{\mathrm{Ca}}$ for bistable conduction detected in a wide range of parameters are well within the experimentally determined activation thresholds of the known $\mathrm{Na}^{+}$and $\mathrm{Ca}^{2+}$ channel families. Since the bistable conduction is mediated by the $\mathrm{I}_{\mathrm{Na}}$ alone in the $\mathrm{HH}$ model, it is likely a generic mechanism applicable to 
conduction not only in the never systems, but also in other electrically excitable media, such as cardiac muscles.

\section{Results}

\section{Bistable conduction in the cable equation}

To observe bistable conduction in the cable equation with the $\mathrm{HH}$ model ${ }^{17}$, we change some of the parameters of the model. To examine the role of $\mathrm{I}_{\mathrm{Ca}}$, we add an $\mathrm{I}_{\mathrm{Ca}}$ formulation to the model. The details of the model are presented in Supplemental Information (SI). Figs.1 A and B show a fast wave $(1.4 \mathrm{~m} / \mathrm{s})$ induced by a strong stimulus and a slow wave $(0.21 \mathrm{~m} / \mathrm{s})$ induced by a weak stimulus, respectively. The action potential of the fast wave (inset in Fig.1A) exhibits a steep upstroke, a high amplitude ( $\approx 95 \mathrm{mV}$, from $-65 \mathrm{mV}$ to $30 \mathrm{mV})$, and a short duration $(\approx 10 \mathrm{~ms})$, while that of the slow wave (inset in Fig.1B) exhibits a shallow upstroke, a low amplitude $(\approx 45 \mathrm{mV}$, from -65 to $-20 \mathrm{mV})$, and a long duration $(\approx 40 \mathrm{~ms})$. These features recapitulate well the experimental observations in the jellyfish (see Fig.S1) and cockroach experiments.

To show how the bistable conduction in the cable is affected by $\mathrm{I}_{\mathrm{Na}}$ and $\mathrm{I}_{\mathrm{Ca}}$, we scan the maximum conductance of $\mathrm{I}_{\mathrm{Na}}\left(\mathrm{G}_{\mathrm{Na}}\right)$ and that of $\mathrm{I}_{\mathrm{Ca}}\left(\mathrm{G}_{\mathrm{Ca}}\right)$, and plot the $\mathrm{CV}$ in color in the twoparameter plane (Fig.1C). For each parameter set, a strong stimulus and a weak stimulus as in Figs. $1 \mathrm{~A}$ and B are applied to elicit conduction waves. The gray region in Fig.1C is where bistable conduction occurs. The white region is where both stimuli fail to elicit conduction (conduction failure). The colored regions (I, II, and III) are where both stimuli give rise to a single stable conduction with CV color coded. Regions I and II exhibit stable fast conduction mediated by $\mathrm{I}_{\mathrm{Na}}$, and region III exhibits stable slow conduction mediated by $\mathrm{I}_{\mathrm{Ca}}$. The presence of $\mathrm{I}_{\mathrm{Ca}}$ promotes bistable conduction, however, $\mathrm{I}_{\mathrm{Ca}}$ is not required since the bistable conduction occurs in the absence of $\mathrm{I}_{\mathrm{Ca}}\left(\mathrm{G}_{\mathrm{Ca}}=0\right)$. In the absence of $\mathrm{I}_{\mathrm{Ca}}\left(\mathrm{G}_{\mathrm{Ca}}=0\right)$, bistable conduction occurs in an intermediate range of $\mathrm{G}_{\mathrm{Na}}$, and the fast conduction occurs when $\mathrm{G}_{\mathrm{Na}}$ is either above or below this range. Therefore, bistable conduction can occur in the $\mathrm{HH}$ model mediated by $\mathrm{I}_{\mathrm{Na}}$ alone without requiring the presence of $I_{\mathrm{Ca}}$ or another inward current. In Fig.1D, we plot CV versus $\mathrm{G}_{\mathrm{Na}}$ for a fixed $\mathrm{G}_{\mathrm{Ca}}$, showing a typical hysteresis, a hallmark of bistability.

\section{Mechanism of bistable conduction}

Since bistable conduction can occur in the absence of $\mathrm{I}_{\mathrm{Ca}}$, we investigate how it occurs in in the original $\mathrm{HH}$ model, focusing on the role of $\mathrm{I}_{\mathrm{Na}}$. Fig. $2 \mathrm{~A}$ shows a fast wave induced by a stimulus slightly above the critical strength and a slow wave by a stimulus slightly below the critical strength in the absence of $\mathrm{I}_{\mathrm{Ca}}$. In the first $40 \mathrm{~ms}$, the action potentials are almost identical for the two cases, which then bifurcate (as indicated by the arrow) into a stable fast wave and a stable slow wave. Since the stimuli are very close to the critical strength, the two waves in the first $40 \mathrm{~ms}$ are close to the solution of the unstable conduction. The open circles in Fig. 1D are calculated from the unstable conduction. In the three conduction behaviors, besides the difference in the amplitude and duration of the action potentials, another important difference is the upstroke speed of the wavefront. For the degeneration from the unstable conduction to the stable fast wave, the upstroke speed of the wavefront becomes faster and faster until reaching the steady-state conduction. For the degeneration from the unstable conduction to the stable slow wave, the 
upstroke speed of the wavefront becomes slower and slower until reaching the steady-state conduction. Because of the different upstroke speeds, the inactivation of $\mathrm{I}_{\mathrm{Na}}$ is different. In Fig.2B, we plot the steady-state inactivation curve of $\mathrm{I}_{\mathrm{Na}}\left(\mathrm{h}_{\infty}\right.$, thick green) and the trajectories of the three types of conduction in the V-h plane. The trajectory of the steady-state slow wave (thick red) undergoes a path that is very close to the steady-state inactivation curve, while the trajectory of the steady-state fast wave (thick blue) undergoes a path that is far away from the steady-state curve. The thin red and blue trajectories are the transient ones degenerating from the steady-state unstable wave (dashed) to the two stable waves, respectively. In other words, the steady-state unstable trajectory (dashed) is the separatrix of the two stable waves.

As indicated by the two cases above, a slower upstroke speed causes more $\mathrm{I}_{\mathrm{Na}}$ inactivation, which in turn results in an even slower upstroke in the next conduction site, or vice versa, forming a positive feedback loop. Therefore, the formation of the stable fast and slow conduction in the cable is a result of the positive feedback in upstroke speed of the wavefront mediated by $\mathrm{I}_{\mathrm{Na}}$ inactivation, result in two modes of $\mathrm{I}_{\mathrm{Na}}$ activation/inactivation. This causes bistable conduction to occur in the intermediate range of $\mathrm{G}_{\mathrm{Na}}$. When $\mathrm{G}_{\mathrm{Na}}$ is too large, the high $\mathrm{I}_{\mathrm{Na}}$ mode is always activated and thus only the fast wave occurs. When $\mathrm{G}_{\mathrm{Na}}$ is too small, the low $\mathrm{I}_{\mathrm{Na}}$ is not large enough to support the slow wave and thus only the high $\mathrm{I}_{\mathrm{Na}}$-mediated fast wave is observed. However, $\mathrm{I}_{\mathrm{Ca}}$ can rescue the slow wave and thus extends the bistable conduction in a wider parameter space (Fig.1C).

To further understand the mechanism of bistable conduction, we obtain an analytical solution using a two-variable model which includes only $\mathrm{I}_{\mathrm{Na}}$ and $\mathrm{I}_{\mathrm{L}}$, i.e.,

$$
\begin{aligned}
& \frac{\partial \mathrm{V}}{\partial \mathrm{t}}=\frac{\partial^{2} \mathrm{~V}}{\partial \mathrm{x}^{2}}+\sigma_{\mathrm{Na}} \mathrm{m}_{\infty}(\mathrm{V}) \mathrm{h}-\mathrm{G}_{\mathrm{L}}\left(\mathrm{V}-\mathrm{E}_{\mathrm{L}}\right) \\
& \frac{\partial \mathrm{h}}{\partial \mathrm{t}}=\frac{\mathrm{h}_{\infty}(\mathrm{V})-\mathrm{h}}{\tau_{h}}
\end{aligned}
$$

where $V$ is voltage and $h$ the inactivation gating variable of $\mathrm{I}_{\mathrm{Na}} \cdot \sigma_{\mathrm{Na}}$ is a parameter proportional to $\mathrm{G}_{\mathrm{Na}} . m_{\infty}(V)$ and $h_{\infty}(V)$ are Heaviside functions:

$$
m_{\infty}(V)= \begin{cases}0, & V<V_{m} \\ 1, & V \geq V_{m}\end{cases}
$$

and

$$
h_{\infty}(V)= \begin{cases}1, & V<V_{h} \\ h_{0}, & V \geq V_{h}\end{cases}
$$

By using the moving coordinate system and continuation conditions, one can obtain that the conduction velocity c satisfies the following equation (see SI method for detailed derivation):

$$
-\left(\mathrm{V}_{\mathrm{m}}-\mathrm{E}_{\mathrm{L}}\right) \sqrt{c^{2}+4 G_{L}}+\sigma_{\mathrm{Na}}\left[\left(\beta+\frac{h_{0}}{\mathrm{G}_{\mathrm{L}}}\right) \delta-\frac{\beta}{\tau_{h} \mathrm{c}}\right]=0
$$

where $\delta=\frac{-\mathrm{c}+\sqrt{\mathrm{c}^{2}+4 \mathrm{G}_{\mathrm{L}}}}{2}, \beta=\frac{\left(1-\mathrm{h}_{0}\right) \mathrm{e}^{\frac{-\varepsilon}{\tau_{h}}}}{\mathrm{G}_{\mathrm{L}}-\frac{1}{\left(\tau_{h} \mathrm{c}\right)^{2}}-\frac{1}{\tau_{h}}}$, and $\varepsilon=\frac{\ln \left(\frac{\mathrm{V}_{\mathrm{h}}-\mathrm{E}_{\mathrm{L}}}{\mathrm{V}_{\mathrm{m}}-\mathrm{E}_{\mathrm{L}}}\right)}{-c-\delta}$. One can rewrite Eq.5 in the form as $\sigma_{\mathrm{Na}}=f\left(c, \tau_{h}, V_{m}, V_{h}, h_{0}, G_{L}, E_{L}\right)$, i.e., $\sigma_{\mathrm{Na}}$ is expressed as a nonlinear function of c. Fig.3A plots c versus $\sigma_{\mathrm{Na}}$ calculated using this formulation with other parameters fixed, showing that $\mathrm{c}$ is bistable 
in a certain range of $\sigma_{\mathrm{Na}}$. One can also numerically solve Eq.5 to obtain c when the parameters are given. Fig.3B shows the conduction behaviors versus $\sigma_{\mathrm{Na}}$ and $\tau_{\mathrm{h}}$, showing that the bistable region decreases as $\tau_{\mathrm{h}}$. We perform additional simulations of the $1 \mathrm{D}$ cable with the $\mathrm{HH}$ model to investigate the effects of $\mathrm{I}_{\mathrm{Na}}$ kinetics on the conduction behaviors. Fig.3C shows the conduction behaviors versus $\tau_{\mathrm{h}}$ and $\tau_{\mathrm{m}}$ (activation time constant of $\mathrm{I}_{\mathrm{Na}}$ ) and Fig.3D shows the conduction behaviors versus $G_{\mathrm{Na}}$ and $\tau_{\mathrm{h}}$. Note that the phase diagram in Fig.3D is similar to that in Fig.3B, indicating that theoretical predictions agree well with the numerical simulation results of the $\mathrm{HH}$ model.

\section{Robustness of bistable conduction}

In the case of Aglantha digitale ${ }^{13}$, based on their observation that the fast wave is mediated by $\mathrm{I}_{\mathrm{Na}}$ and the slow wave by $\mathrm{I}_{\mathrm{Ca}}$, the authors hypothesized that to facilitate the fast and slow conduction in the same axon, the $\mathrm{Na}^{+}$channel activation threshold is much higher than the $\mathrm{Ca}^{2+}$ channel activation threshold so that during $\mathrm{I}_{\mathrm{Ca}}$-mediated conduction (the slow wave), the peak voltage remains low enough to avoid activation of $\mathrm{I}_{\mathrm{Na}}$. On the other hand, in the case of cockroach experiments ${ }^{14}$, since the slow wave was still mediated by $\mathrm{I}_{\mathrm{Na}}$, the authors then hypothesized that there might exist another type of $\mathrm{I}_{\mathrm{Na}}$ with a low activation threshold in the same axon to explain their observations. In other words, theses authors hypothesized that the fast and slow conduction are mediated separately by two inward currents with a certain required difference in activation thresholds (we call it dual-threshold hypothesis). In the original $\mathrm{HH}$ model, since there is only one inward current (i.e., $\mathrm{I}_{\mathrm{Na}}$ ), it can only exhibit a single-threshold response (Fig.4A). On the other hand, after adding $\mathrm{I}_{\mathrm{Ca}}$ to the $\mathrm{HH}$ model, it can exhibit a dual-threshold response in which two types of action potentials occur depending on the stimulus strength (Fig.4B). Since there are two distinct types of action potentials, one would expect that each type will give rise to a conduction in the cable, resulting in stimulus-dependent fast and slow conduction. This raises a question: which of the two mechanisms is robust or more likely to occur in the real systems?

To address this question, we perform the following investigations. We first carry out a large number of simulations of the cable equation with randomly selected parameters in assigned intervals (see details in $S I$ ). We detect the parameter sets giving rise to both fast and slow conduction. We then use these parameter sets to perform single-cell simulations to filter out the single-threshold and dual-threshold responses by examining the stimulus-response relationship as shown in Figs.4 A and B. The parameter sets (open dots) exhibiting single-threshold responses are plotted in Fig.4C and those exhibiting dual-threshold responses in Fig.4D. The data points are plotted on the plane of the half-activation voltages for $\mathrm{Na}^{+}$channels $\left(\mathrm{V}_{\mathrm{Na}, 1 / 2}\right)$ and $\mathrm{Ca}^{2+}$ channels $\left(\mathrm{V}_{\mathrm{Ca}, 1 / 2}\right)$. See $S I$ for definitions and ranges of $\mathrm{V}_{\mathrm{Na}, 1 / 2}$ and $\mathrm{V}_{\mathrm{Ca}, 1 / 2}$ in the model. We also plot the experimentally measured $\mathrm{V}_{\mathrm{Na}, 1 / 2}$ (red squares) and $\mathrm{V}_{\mathrm{Ca}, 1 / 2}$ (green circles) surveyed from literature by Catterall et al ${ }^{18,19}$. $\mathrm{V}_{\mathrm{Na}, 1 / 2}$ for the known 10 members of the $\mathrm{Na}^{+}$channel family ranges from $56 \mathrm{mV}$ to $-8.8 \mathrm{mV}^{18}$, and $\mathrm{V}_{\mathrm{Ca}, 1 / 2}$ for the 10 members of the $\mathrm{Ca}^{2+}$ channel family ranges from -46 $\mathrm{mV}$ to $14 \mathrm{mV}^{19}$. $\mathrm{V}_{\mathrm{Na}, 1 / 2}$ and $\mathrm{V}_{\mathrm{Ca}, 1 / 2}$ are plotted in a way so that all possible combinations of the 10 members of the $\mathrm{Na}^{+}$channel family and the 10 members of the $\mathrm{Ca}^{2+}$ channel family fall inside the dashed red box. Therefore, we argue that the parameter sets fall inside the box are the ones that can occur in the real system. 
The data sets exhibiting single-threshold response fall both inside and outside the dashed box (Fig.4C). We plot the distributions of $\mathrm{CV}$ for the fast and slow conduction in Fig.4E, and the ratios of the fast-to-slow $\mathrm{CV}$ in Fig.3F. The average slow $\mathrm{CV}$ is $0.23 \pm 0.08 \mathrm{~m} / \mathrm{s}$ and the average fast $\mathrm{CV}$ is $1.10 \pm 0.32 \mathrm{~m} / \mathrm{s}$. The average of the fast-to-slow $\mathrm{CV}$ ratio is $7.0 \pm 7.84$. The fast-to-slow $\mathrm{CV}$ ratios were 5 to 10 in the experimentally measured CVs in the giant axons of Aglantha digitale 13 and cockroaches ${ }^{14}$, which are in the same range as obtained in the simulations. Therefore, the bistable conduction mechanism agrees well with experimental data and is robust.

The data sets exhibiting dual-threshold response fall into two groups (Fig.4D). One group is completely outside the box (lower right), which occurs for $\mathrm{V}_{\mathrm{Na}, 1 / 2}$ is $40 \mathrm{mV}$ higher than $\mathrm{V}_{\mathrm{Ca}, 1 / 2}$. The other group can still fall into the box (upper left), which occurs for $V_{C a, 1 / 2}$ is $30 \mathrm{mV}$ higher than $\mathrm{V}_{\mathrm{Na}, 1 / 2}$. However, some caveats of this group of data are worth noting. The slow wave is mediated by $\mathrm{I}_{\mathrm{Na}}$ and the fast wave by $\mathrm{I}_{\mathrm{Ca}}$, which is not what occurs in either the jellyfish ${ }^{13}$ or the cockroaches ${ }^{14}$. Although the data sets were filtered through the dual-threshold criterion in single cells, when we check the behaviors in the cable, the $\mathrm{Na}^{+}$channel exhibit similar response as in Fig.2, indicating that the mechanism may still be bistable conduction as in the single-threshold case. For example, when we remove $\mathrm{I}_{\mathrm{Ca}}$ from the data sets in Fig.4D, we can still observe bistable conduction. To show this, we perform the same simulations using the parameter sets in Fig.4D without $\mathrm{I}_{\mathrm{Ca}}$ (See Fig.S2A), the parameter sets outside the box disappear but about $50 \%$ of the parameter sets inside the box retain. Another evidence to support this is that if we slow the $\mathrm{Na}^{+}$ channel inactivation, this group of parameter sets will disappear. We perform the same simulations as in Fig.4 except that the inactivation is slowed by increasing $\gamma\left(\tau_{h}\right)=0.35$ to $\gamma\left(\tau_{h}\right)=1$ (see Fig.S2B). The upper group disappears, but the lower group retains and is still outside the dashed box. The results shown in Fig.4 and Fig.S2 indicate that the dual-threshold mechanism is difficult to be satisfied in the real systems.

In the model, the resting potential is set at $-65 \mathrm{mV}$. If we lower the resting potential, e.g., to $-75 \mathrm{mV}$, we observe almost the same results (Fig.S3) as those shown in Figs. 4 and S2 except that the minimum $\mathrm{I}_{\mathrm{Na}}$ or $\mathrm{I}_{\mathrm{Ca}}$ activation threshold for bistable conduction has a roughly $10 \mathrm{mV}$ shift due to the lowering of the resting potential.

\section{Discussion}

Nerve conduction can be well described by the cable equation with the $\mathrm{HH}$ model ${ }^{5-9}$. It is well known that the cable equation with FitzHug-Nagumo model or the HH model exhibit monostable conduction ${ }^{10}$. In this study, we show that the cable equation with the HH model can exhibit bistable conduction in which a fast and a slow stable conduction occur in the same cable depending on the stimulus strength. The bistable behavior is a result of the positive feedback of the wavefront upstroke speed mediated by the $\mathrm{Na}^{+}$channel inactivation properties. In other words, this positive feedback cause two stable modes of $\mathrm{I}_{\mathrm{Na}}$ activation, which occurs when the $\mathrm{Na}^{+}$channel inactivation is relatively fast (see also Fig.3). Unlike the fast conduction which occurs as long as the $\mathrm{G}_{\mathrm{Na}}$ is greater than a critical value, bistable conduction can only occur in an intermediate range of $\mathrm{G}_{\mathrm{Na}}$. This is because when $\mathrm{G}_{\mathrm{Na}}$ is too large, the high $\mathrm{I}_{\mathrm{Na}}$ mode is always activated and thus only the fast wave can occur. When $\mathrm{G}_{\mathrm{Na}}$ is too small, the low $\mathrm{I}_{\mathrm{Na}}$ cannot support a stable slow conduction, and thus only the fast conduction can occur. However, the failed slow conduction can be rescued by the addition of $\mathrm{I}_{\mathrm{Ca}}$, which can substantially extend the bistable conduction regime 
(see Fig.1C). We use an analytical treatment of simplified cable model to demonstrate that bistable conduction can be mediated a single inward current, namely $\mathrm{I}_{\mathrm{Na}}$. Using simulations with randomly drawing parameter sets, we also show that the bistable conduction is robust, which can occur well within the experimentally determined ranges of the activation thresholds of the known $\mathrm{Na}^{+}$and $\mathrm{Ca}^{2+}$ channel families.

Our computer simulation results agree well with the experimental observations of electrical conduction in the giant axons of jellyfish and cockroaches. For example, the action potential profiles in the fast and slow waves and the ratios of the fast-to-slow $\mathrm{CV}$ in the simulations agree with those shown in experiments in both jellyfish and cockroaches. More importantly, our theoretical study unifies the seemly different experimental observations in jellyfish and cockroaches to the same general mechanism. In the jellyfish experiments, Mackie and Meech ${ }^{13}$ showed that the slow wave was blocked by $\mathrm{Ca}^{2+}$ channel blockers, and thus concluded that the slow wave was mediated by $I_{C a}$. This led them to hypothesize that the activation threshold of $\mathrm{I}_{\mathrm{Na}}$ has to be much higher than that of $\mathrm{I}_{\mathrm{Ca}}$ to allow the two waves to occur. As shown in our simulations (Fig.1C), although $\mathrm{I}_{\mathrm{Ca}}$ is not required for bistable conduction, it potentiates bistable conduction by rescuing the slow conduction. Therefore, blocking it will suppress the slow conduction, agreeing with the experimental observation. On the other hand, in the cockroach experiments, Hochner and Spira ${ }^{14}$ found that the slow wave was blocked not by $\mathrm{Ca}^{2+}$ channel blockers but by $\mathrm{Na}^{+}$channel blockers, differing from the jellyfish experiments. They then hypothesized that a low-threshold $\mathrm{I}_{\mathrm{Na}}$ must be responsible for the slow wave. Our simulations showed that $\mathrm{I}_{\mathrm{Ca}}$ or another low-threshold $\mathrm{I}_{\mathrm{Na}}$ is not required since a single $\mathrm{I}_{\mathrm{Na}}$ could produce both the slow and fast conduction via the bistable dynamics. In other words, the fast and slow conduction observed in cockroaches may originated from the same $\mathrm{I}_{\mathrm{Na}}$. A moderate $\mathrm{I}_{\mathrm{Na}}$ reduction may block the slow wave but not the fast wave while a strong $\mathrm{I}_{\mathrm{Na}}$ reduction can block both waves (see Fig.1C). Therefore, the experimental observations in both jellyfish and cockroaches can be explained by the same mechanism in different parameter regimes.

Besides the giant axons in jellyfish and cockroaches, bistable conduction may also occur in other nerve systems. For example, it was shown that the spontaneous waves and the evoked waves exhibit distinct CVs in the same rat visual cortex ${ }^{15,16}$, which could be a result of bistable conduction. In addition, bimodal CV distributions were widely observed in sensory and motor nerves ${ }^{20-26}$, which were traditionally attributed to the size difference of the fibers. However, it was also shown that the action potential profiles in the fast conduction are different from those in the slow conduction, i.e., the amplitude is much lower and the duration is much longer in the slow conduction ${ }^{20}$, similar to those observed in the jellyfish and cockroaches. The large difference in action potential properties cannot be simply attributed to the size difference of the nerve fibers since although the size of the nerve fibers can largely affect $\mathrm{CV}$, it may only have a small effect on the action potential properties. Bistable conduction can be a candidate mechanism for bimodal CV distributions, which needs to be verified in future experimental studies.

Finally, as shown in our simulations (Fig. 4), the dual-threshold mechanism is theoretically plausible, however, it requires a very large minimum difference in the activation thresholds of $\mathrm{I}_{\mathrm{Na}}$ and $\mathrm{I}_{\mathrm{Ca}}$, which may not be satisfied easily in the real systems. On the other hand, the bistable conduction mechanism can be easily achieved without requiring any gap or correlation between the activation thresholds of the two types of ionic currents. Moreover, the bistable conduction 
mechanism may also have an evolutionary advantage over the dual-threshold mechanism since a single rather than two inward currents can provide two survival functions as in the jellyfish. Although it is unclear what are the functional roles of the fast and slow conduction in other species or diseases, as it is well known that bistability is a ubiquitous phenomenon in biology and responsible for many biological functions ${ }^{27-30}$, we believe that bistable conduction may also play important roles in nerve functions under health and diseased conditions. As shown in our study, the bistable conduction occurs in the cable equation with the $\mathrm{HH}$ model, we believe that it is a generic mechanism that is applicable to not only the nerve systems but also other electrically excitable tissue, such as cardiac muscles.

\section{Methods}

The HH model ${ }^{17}$ with modifications is used to simulate action potential conduction in a cable with the following partial differential equation for voltage $(V)$ :

$$
\frac{\partial V}{\partial t}=-\frac{I_{\text {ion }}+I_{\text {stim }}}{C_{m}}+D \frac{\partial^{2} V}{\partial x^{2}}
$$

where $C_{\mathrm{m}}=1 \mu \mathrm{F} / \mathrm{cm}^{2}$ is the membrane capacitance, and $\mathrm{D}=0.0045 \mathrm{~cm}^{2} / \mathrm{ms}$ is the diffusion constant. $I_{\text {stim }}$ is the stimulus current density and $I_{\text {ion }}$ is the total ionic current density consisting of different types of ionic currents, i.e.,

$$
I_{\text {ion }}=I_{N a}+I_{C a}+I_{K}+I_{L} \text {. }
$$

$I_{N a}$ is the $\mathrm{Na}^{+}$current, $I_{C a}$ is the $\mathrm{Ca}^{2+}$ current, $\mathrm{I}_{\mathrm{K}}$ is the $\mathrm{K}^{+}$current, and $\mathrm{I}_{\mathrm{L}}$ is the leak current. The formulations of $\mathrm{I}_{\mathrm{Na}}, \mathrm{I}_{\mathrm{K}}$, and $\mathrm{I}_{\mathrm{L}}$ are the same as in the $\mathrm{HH}$ model. $\mathrm{I}_{\mathrm{Ca}}$ is formulated based on Medlock et al ${ }^{31}$ with the addition of an inactivation gate. Eq. 1 is numerically solved using a forward Euler method with $\Delta x=0.045 \mathrm{~cm}$ and $\Delta t=0.005 \mathrm{~ms}$. The gating variables are integrated using the method by Rush and Larsen ${ }^{32}$. No-flux boundary condition is used. The mathematical details of the model and numerical algorithms are presented in SI.

\section{Funding Sources}

This study was supported by National Institutes of Health grants R01 HL134709 and R01 HL139829.

\section{Author contributions}

ZQ conceived the project, supervised the research, provided funding, and wrote the manuscript; ZZ performed the simulations and mathematical analyses; ZQ and ZZ analyzed the results and edited the manuscript.

\section{References}

1 Castelfranco, A. M. \& Hartline, D. K. Evolution of rapid nerve conduction. Brain Research 1641, 11-33 (2016).

2 Hursh, J. B. Conduction velocity and diameter of nerve fibers. American Journal of Physiology 127, 131-139 (1939).

3 Waxman, S. G. Determinants of conduction velocity in myelinated nerve fibers. Muscle \& Nerve 3, 141-150 (1980).

4 Rosenthal, J. \& Bezanilla, F. Seasonal variation in conduction velocity of action potentials in squid giant axons. The Biological Bulletin 199, 135-143 (2000). 
5 Cooley, J. W. \& Dodge, F. A. Digital Computer Solutions for Excitation and Propagation of the Nerve Impulse. Biophysical Journal 6, 583-599 (1966).

6 Goldman, L. \& Albus, J. S. Computation of Impulse Conduction in Myelinated Fibers; Theoretical Basis of the Velocity-Diameter Relation. Biophysical Journal 8, 596-607 (1968).

7 Parnas, I. \& Segev, I. A mathematical model for conduction of action potentials along bifurcating axons. The Journal of Physiology 295, 323-343 (1979).

8 Miller, R. N. \& Rinzel, J. The dependence of impulse propagation speed on firing frequency, dispersion, for the Hodgkin-Huxley model. Biophysical Journal 34, 227-259 (1981).

9 Young, R. G., Castelfranco, A. M. \& Hartline, D. K. The "Lillie transition": models of the onset of saltatory conduction in myelinating axons. J Comput Neurosci 34, 533-546 (2013).

10 Keener, J. P. \& Sneyd, J. Mathematical Physiology. (Springer, 1998).

11 Satterlie, R. in Oxford Research Encyclopedia, Neuroscience (oxfordre.com/neuroscience) (Oxford University Press, 2018).

12 Meech, R. W. in Oxford Research Encyclopedia, Neuroscience (oxfordre.com/neuroscience) (Oxford University Press, 2019).

13 Mackie, G. O. \& Meech, R. W. Separate sodium and calcium spikes in the same axon. Nature 313, 791-793 (1985).

14 Hochner, B. \& Spira, M. E. Two distinct propagating regenerative potentials in a single ethanol-treated axon. Brain Research 398, 164-168 (1986).

$15 \mathrm{Xu}, \mathrm{W} .$, Huang, X., Takagaki, K. \& Wu, J.-Y. Compression and Reflection of Visually Evoked Cortical Waves. Neuron 55, 119-129 (2007).

16 Han, F., Caporale, N. \& Dan, Y. Reverberation of recent visual experience in spontaneous cortical waves. Neuron 60, 321-327 (2008).

17 Hodgkin, A. L. \& Huxley, A. F. A Quantitative Description of Membrane Current and Its Application to Conduction and Excitation in Nerve. Journal of Physiology 117, 500-544 (1952).

18 Catterall, W. A., Goldin, A. L. \& Waxman, S. G. International Union of Pharmacology. XLVII. Nomenclature and Structure-Function Relationships of Voltage-Gated Sodium Channels. Pharmacological Reviews 57, 397-409 (2005).

19 Catterall, W. A., Perez-Reyes, E., Snutch, T. P. \& Striessnig, J. International Union of Pharmacology. XLVIII. Nomenclature and Structure-Function Relationships of VoltageGated Calcium Channels. Pharmacological Reviews 57, 411-425 (2005).

20 Andrew, B. L. \& Part, N. J. Properties of fast and slow motor units in hind limb and tail muscles of the rat. Q J Exp Physiol Cogn Med Sci 57, 213-225 (1972).

21 Calvin, W. H. \& Sypert, G. W. Fast and slow pyramidal tract neurons: an intracellular analysis of their contrasting repetitive firing properties in the cat. Journal of Neurophysiology 39, 420-434 (1976).

22 Cummins, K. L., Dorfman, L. J. \& Perkel, D. H. Nerve fiber conduction-velocity distributions. II. Estimation based on two compound action potentials. Electroencephalography and Clinical Neurophysiology 46, 647-658 (1979).

23 Milner, T. E. \& Stein, R. B. The effects of axotomy on the conduction of action potentials in peripheral sensory and motor nerve fibres. Journal of Neurology, Neurosurgery \&amp; Psychiatry 44, 485-496 (1981). 
24 Todnem, K., Knudsen, G., Riise, T., Nyland, H. \& Aarli, J. A. The non-linear relationship between nerve conduction velocity and skin temperature. J Neurol Neurosurg Psychiatry 52, 497-501 (1989).

25 van Veen, B. K., Schellens, R. L. L. A., Stegeman, D. F., Schoonhoven, R. \& GabreëlsFesten, A. A. W. M. Conduction velocity distributions compared to fiber size distributions in normal human sural nerve. Muscle \& Nerve 18, 1121-1127 (1995).

$26 \mathrm{Ni}$, Z. et al. Measuring conduction velocity distributions in peripheral nerves using neurophysiological techniques. Clinical Neurophysiology 131, 1581-1588 (2020).

27 Gardner, T. S., Cantor, C. R. \& Collins, J. J. Construction of a genetic toggle switch in Escherichia coli. Nature 403, 339-342 (2000).

28 Sha, W. et al. Hysteresis drives cell-cycle transitions in Xenopus laevis egg extracts. Proc Natl Acad Sci U S A 100, 975-980. (2003).

29 Xiong, W. \& Ferrell, J. E., Jr. A positive-feedback-based bistable 'memory module' that governs a cell fate decision. Nature 426, 460-465 (2003).

30 Qu, Z., Hu, G., Garfinkel, A. \& Weiss, J. N. Nonlinear and stochastic dynamics in the heart. Phys Rep 543, 61-162 (2014).

31 Medlock, L., Shute, L., Fry, M., Standage, D. \& Ferguson, A. V. Ionic mechanisms underlying tonic and burst firing behavior in subfornical organ neurons: a combined experimental and modeling study. J Neurophysiol 120, 2269-2281 (2018).

32 Rush, S. \& Larsen, H. A practical algorithm for solving dynamic membrane equations. IEEE Trans. Biomed. Eng. 25, 389-392 (1978). 

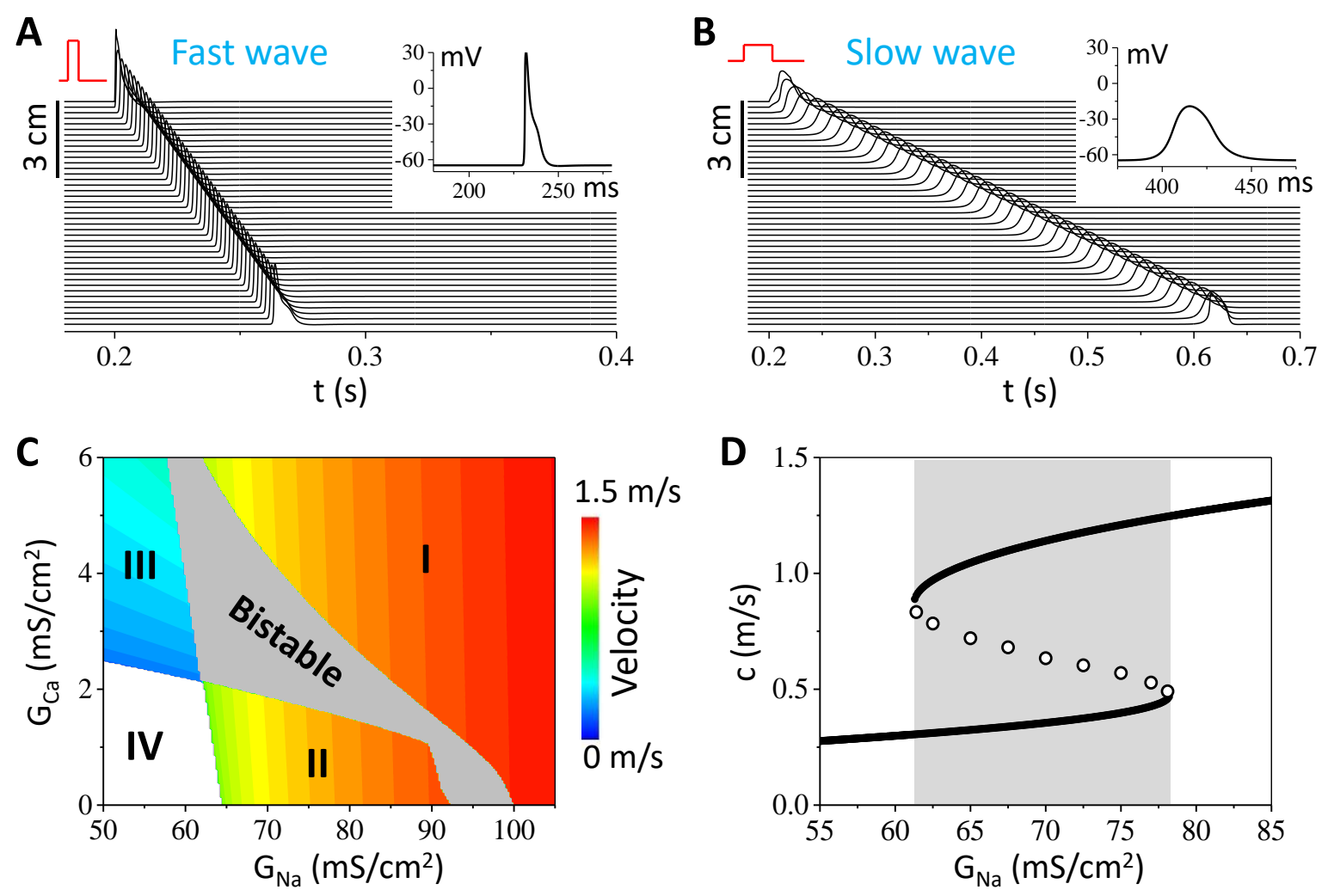

Figure 1. Bistable conduction in the cable equation. A and B. Stimulus-dependent fast (A) and slow (B) conduction in the same cable. The stimulus in A is $200 \mu \mathrm{A} / \mathrm{cm}^{2}$ with a $0.5 \mathrm{~ms}$ duration while the stimulus in $\mathrm{B}$ is $5 \mu \mathrm{A} / \mathrm{cm}^{2}$ with a $20 \mathrm{~ms}$ duration. The stimulus is applied to the first $0.225 \mathrm{~cm}$ of the cable. Insets show action potentials from the middle of the cable for the two cases. $\mathrm{G}_{\mathrm{Na}}=95 \mathrm{mS} / \mathrm{cm}^{2}$ and $\mathrm{G}_{\mathrm{Ca}}=0 \mathrm{mS} / \mathrm{cm}^{2}$. C. Phase diagram showing conduction behaviors in the $\mathrm{G}_{\mathrm{Na}}$ and $\mathrm{G}_{\mathrm{Ca}}$ plane. Regions I and II are monostable fast conduction, region III is monostable slow conduction, region IV is conduction failure, and the gray region is bistable conduction. The phase diagram is obtained using the strong and weak stimulus protocols as in A and B. D. CV versus $\mathrm{G}_{\mathrm{Na}}$ for $\mathrm{G}_{\mathrm{Ca}}=3 \mathrm{mS} / \mathrm{cm}^{2}$. Solid circles are stable conduction and open circles are unstable conduction (the saddle points). The saddle points are determined by a stimulus very close to the critical stimulus (see Fig.2A for an example). The gray marks the bistable region. 

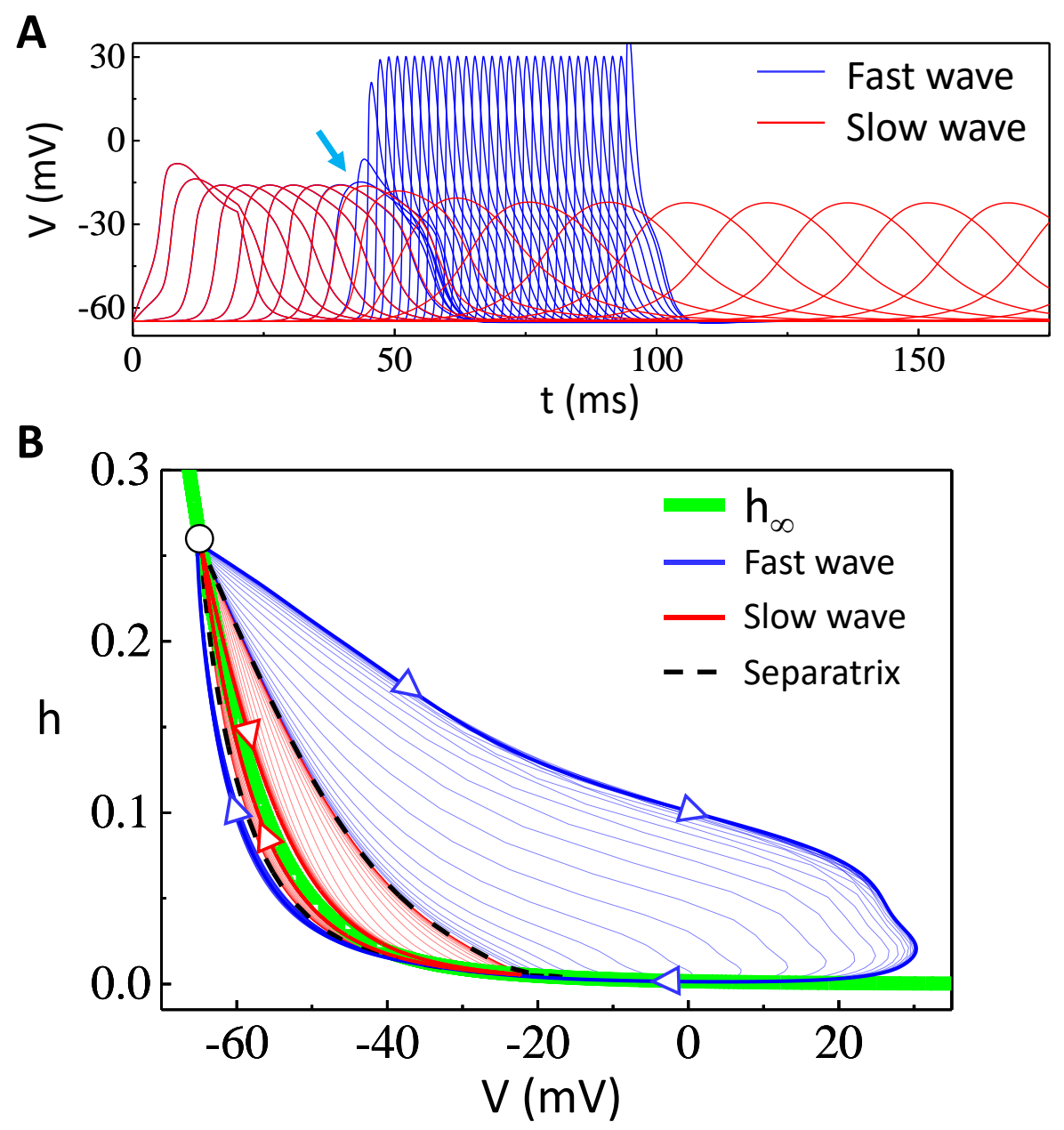

Figure 2. Mechanism of Ina-mediated bistable conduction. A. Formation of stable fast (blue) and slow (red) conduction from the unstable conduction in the absence of $\mathrm{I}_{\mathrm{Ca}}$. The fast wave is induced by a stimulus ( $\mathrm{I}_{\mathrm{stim}}=22.32155910275 \mu \mathrm{A} / \mathrm{cm}^{2}$ with $20 \mathrm{~ms}$ in duration) slightly above the critical strength and the slow wave is by a stimulus $\left(\mathrm{I}_{\mathrm{stim}}=22.32155910270 \mu \mathrm{A} / \mathrm{cm}^{2}\right.$ with $20 \mathrm{~ms}$ in duration) slightly below the critical strength. Voltage traces are recorded at sites $0.225 \mathrm{~cm}$ apart along the cable. The blue and red traces are overlapped until a distance away (marked by the arrow) from the stimulation site. $\mathrm{G}_{\mathrm{Na}}=92 \mathrm{mS} / \mathrm{cm}^{2}$ and $\mathrm{G}_{\mathrm{Ca}}=0 . \mathbf{B} \cdot \mathrm{Na}^{+}$channel inactivation properties during the fast and slow waves. The thick green line is the steady-state inactivation curve of the $\mathrm{Na}^{+}$channel $\left(\mathrm{h}_{\infty}\right)$. The thick red curve with arrows is the trajectory of the steady-state slow wave and the thin red curves are the ones recorded from the transition period from the unstable conduction to the stable slow conduction. The thick blue curve with arrows is the trajectory of the steady-state fast wave and the thin blue curves are the ones recorded from the transition period from the unstable conduction to the stable fast conduction. The black dashed curve is the separatrix of the two types of waves, which is the trajectory taken from the unstable conduction (before the arrow marked in A). 

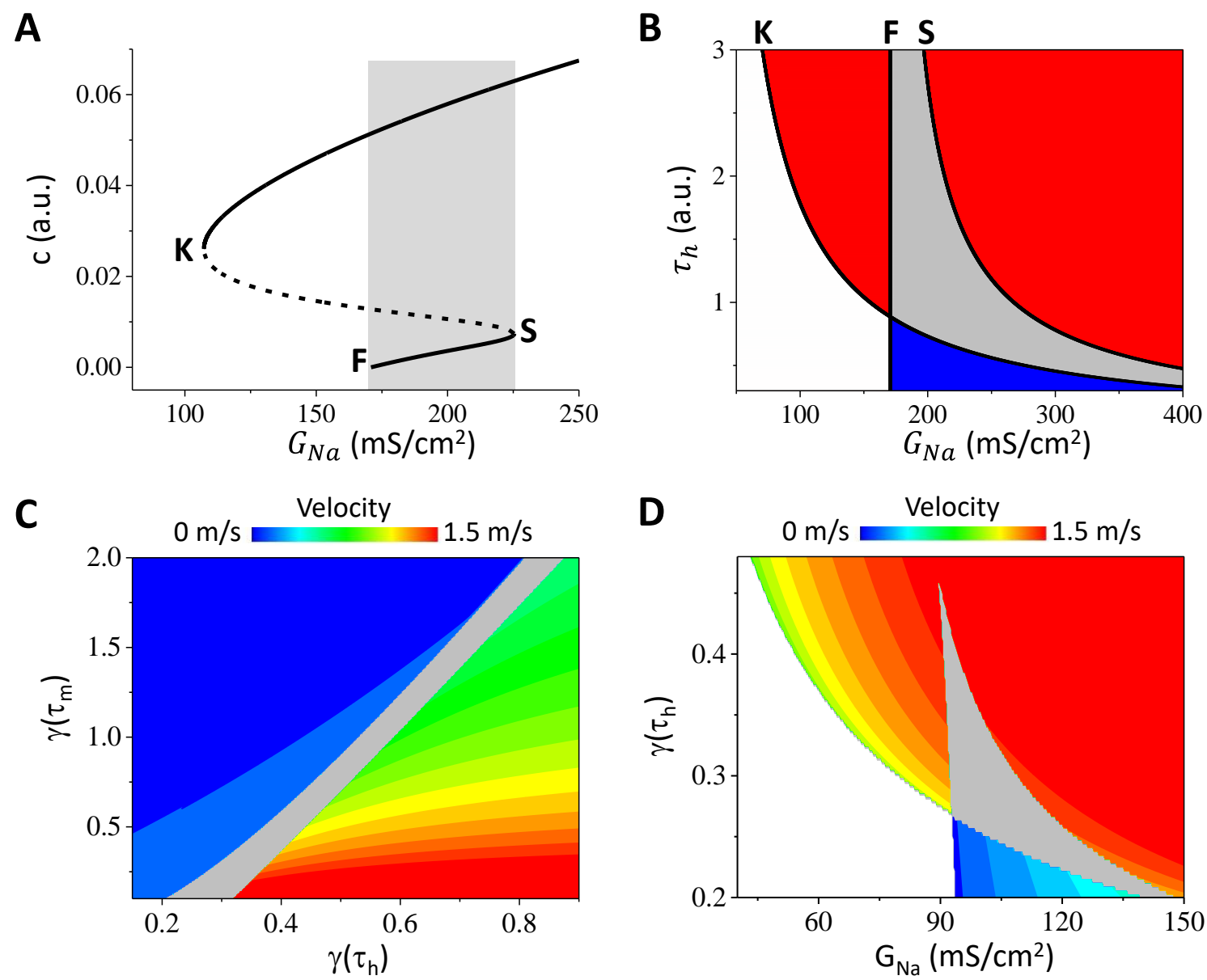

Figure 3. Effects of $I_{\mathrm{Na}}$ kinetics on bistable conduction. A. Conduction velocity c versus $G_{N a}$ calculated from the analytical result Eq.5. K and S mark the saddle-node bifurcation points, and F marks the point of conduction failure of the slow conduction. Solid lines are stable conduction and dashed line is unstable conduction. Gray marks the bistable conduction region. $\tau_{h}=1.6, V_{m}=$ $-19.5 \mathrm{mV}, V_{h}=-55.5 \mathrm{mV}, h_{0}=0.075, G_{L}=0.3, E_{L}=-65 \mathrm{mV}$. We used $\sigma_{N a}=2.1 G_{N a}$ for Eq.5. B. Conduction behaviors versus $G_{N a}$ and $\tau_{h}$ obtained from the analytical result Eq.5. K, F, and $\mathrm{S}$ are the boundaries as marked on $\mathrm{A}$. The gray region is the bistable region. The red regions are monostable fast conduction, the blue region is monostable slow conduction, and the blank region is conduction failure. The parameters are the same as for A. C. Conduction behaviors versus $\gamma\left(\tau_{h}\right)$ and $\gamma\left(\tau_{m}\right)$ from the simulation of the cable equation using the HH model. $(\gamma$ is the fold change of $\tau_{\mathrm{h}}$ or $\tau_{\mathrm{m}}$ from their original values in the HH model, see Eq.S4 in SI). The gray region is the bistable conduction region. $\mathrm{G}_{\mathrm{Na}}=100 \mathrm{mS} / \mathrm{cm}^{2}$ and $\mathrm{G}_{\mathrm{Ca}}=0$. D. Conduction behaviors versus $G_{N a}$ and $\gamma\left(\tau_{h}\right)$ from the simulation of the cable equation using the $\mathrm{HH}$ model. Other parameters were the same as for $\mathrm{C}$. The phase diagrams in $\mathrm{C}$ and $\mathrm{D}$ were obtained and colored the same way as for Fig.1C. 

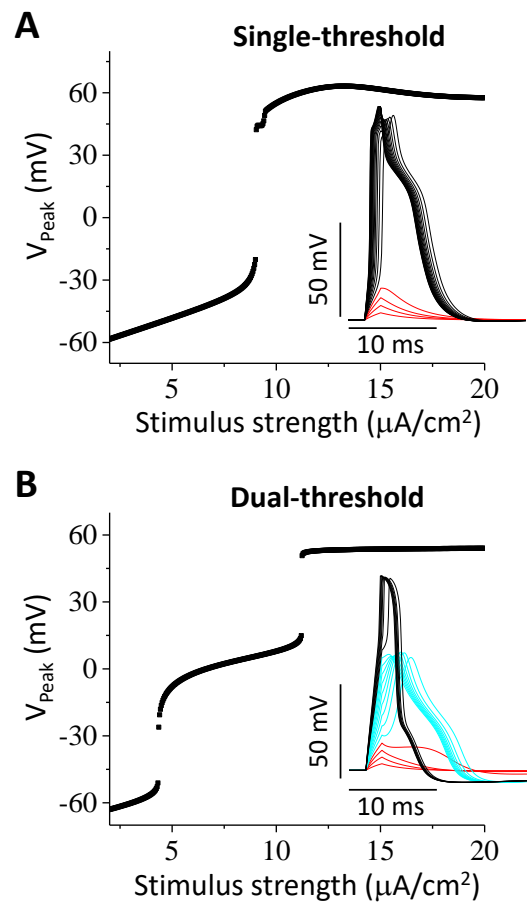
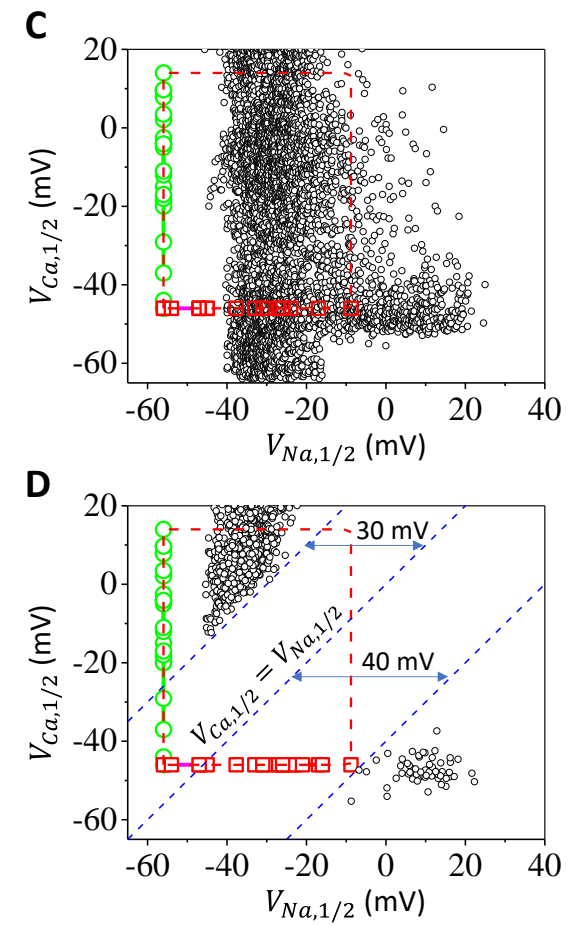

$\mathbf{F}$
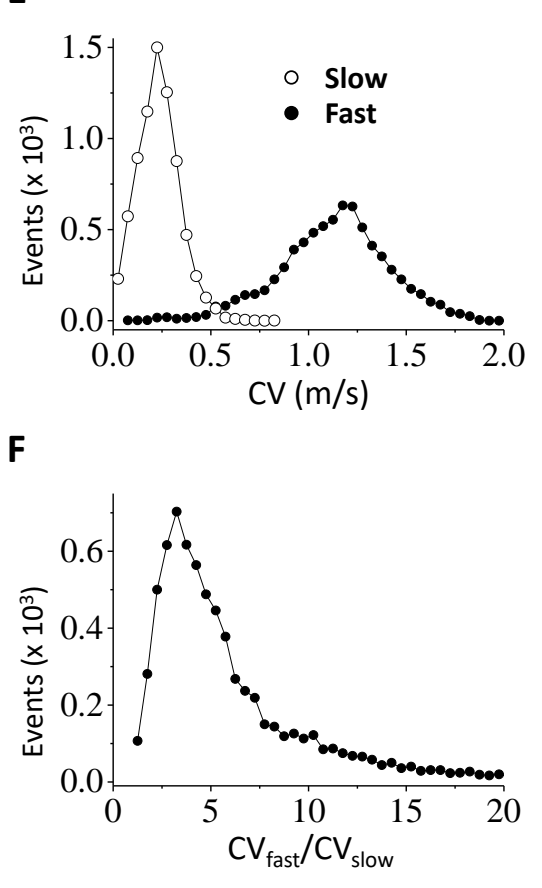

Figure 4. Robustness of bistable conduction. A. Peak voltage versus stimulus strength for a case exhibiting a single threshold. Inset shows the voltage traces for different stimulus strengths. Red traces are the subthreshold responses. B. Same as A but for a case exhibiting dual thresholds. Inset shows that besides the subthreshold response, there are two distinct types of action potentials. $\mathbf{C}$. Parameter sets (black) that give rise to bistable conduction filtered with the single-threshold criterion, plotted in the $\mathrm{V}_{\mathrm{Na}, 1 / 2}$ and $\mathrm{V}_{\mathrm{Ca}, 1 / 2}$ plane. Red squares are $\mathrm{V}_{\mathrm{Na}, 1 / 2}$ taken from Catterall et al 18 and green circles are $\mathrm{V}_{\mathrm{Ca}, 1 / 2}$ taken from Catterall et al ${ }^{19}$. D. Same as $\mathrm{C}$ but for the parameter sets filtered with the dual-threshold criterion. Dashed blue lines are reference lines marking the minimum difference of the two activation thresholds. E. CV distributions for the slow and fast conduction calculated from the data shown in C. F. Distribution of the fast-to-slow CV ratio calculated from the same data in $\mathrm{C}$. 